\title{
Editorial
}

\section{Complications of IVF}

The undaunted revolution in assisted reproductive technologies (ART), such as in vitro fertilization-embryo transfer (IVF-ET), its versatile applications, ever-expanding indications and the promising success rates have made this technology a quick first-line resort for both the clinician and the patient. However, the practice of ART is beset with short and long-term medical complications that may be related to the hormonal (ovarian stimulation) and surgical (transvaginal oocyte recovery) protocols involved, persist for years to come with lethal effects in rare cases, mandating close surveillance.

The short-term complications associated with transvaginal ultrasonography-guided oocyte retrievals include hemorrhage, perforation, intraperitoneal bleeding, rupture of endometriotic cysts, severe to very severe pain that may persist days after the procedure, adnexal torsions, and pelvic infection. Tubo-ovarian abscess is amongst the most common pelvic infection, that may be manifested by abdominal pain, fever and leukocytosis days or months after the procedure, perhaps as an uncommon complication during pregnancy, 1,2 and that may lead to serious complications necessitating hospitalization. Pelvic inflammatory disease, including endometriosis, tubal abnormalities or myomas, overweight, history of abdominal or pelvic surgery, and structural genital anomalies are known risk factors for pelvic abscess during pregnancy. ${ }^{3,2}$ Therapy may include surgery and antibiotics, postpartum percutaneous drainage of the abscess. ${ }^{4}$

The most significant complications of gonadotropin stimulation for ovarian multifollicular development are ovarian hyperstimulation syndrome (OHSS) and the increased risk of multiple pregnancies. OHSS involves significant ovarian hypertrophy associated with the loss of the intravascular fluid to the third space, resulting in ascites, pleural and pericardial effusion, and oliguria, dyspnea with tachypnoe, tachycardia, adult respiratory distress syndrome, renal failure, venous thrombosis, ischemic stroke, and hemorrhage from a ruptured ovary ${ }^{5}$ hypovolemia, electrolyte imbalance and a rise in haematocrit. It may be mild, moderate or severe, may manifest early or late, and the severe form is a lifethreatening. Increased follicular number $\geq 13$ ( $\geq 18$ for severe) and high estradiol (E2) concentrations $(>2,500$ $\mathrm{ng} / \mathrm{L} ;>5,000$ for severe) are significantly predictive of OHSS. Proposed protocols for management include prevention, mild stimulation protocols with small doses of gonadotropin, coasting GnRH antagonist protocol in high-risk patients with low dose hCG triggering, intravenous albumin when given at time of oocyte retrieval, cryopreservation of all embryos (will reduce late-onset OHSS but not early-onset OHSS), in-vitro maturation of oocytes (will avoid ovarian stimulation and totally prevent OHSS). ${ }^{6}$ The increased risk of multiple and pathological multiple pregnancies following multiple embryo transfer, including monozygotic twinning, ectopic and heterotopic pregnancies (combined intrauterine and interstitial twin pregnancies), ${ }^{7}$ and cornual pregnancies, ${ }^{8}$ are serious complications of IVF that warrant early sonographic follow-up as they may be associated with maternal complications (preeclampsia, gestational diabetes, placental abruption, placenta previa and cesarean delivery) and perinatal morbidity (preterm birth, low birth weight) and mortality. Both singleton and twin ART pregnancies may be at higher risk than spontaneous pregnancies for the adverse maternal and perinatal outcomes, necessitating close surveillance during pregnancy. ${ }^{9}$ Spontaneous reductions that occur at $>8$ weeks gestations pose an additional 
obstetric complication.

Long-term complications of gonadotropin stimulation for IVF include thromboembolic disease associated with risk factors as age $\geq 39$ years and mild hyperhomocysteinemia, ${ }^{10}$ possibly breast cancer in women $\geq$ 40 years of age undergoing $\geq 4$ IVF cycles, ${ }^{11}$ and uterine cancer linked to prolonged use of clomiphene citrate (dose $>900 \mathrm{mg}$ ) and/or $\geq 6 \mathrm{IVF}$ cycles, the risk being substantially elevated among women who are both obese and nulligravid. ${ }^{12}$ However, the evidence linking the use of fertility drugs and cancer must be buttressed by larger, adequately powered studies with longer-term follow-up to accurately document the association and employ necessary preventive measures.

Natural/ modified natural cycle IVF and minimal stimulation IVF that aim to decreased the gonadotropin use and duration, the cause of the majority of ART complications, combined with in vitro oocyte maturation and oocyte/embryo cryopreservation by vitrification and elective single embryo transfer, would be the way ahead to avoid the serious complications inflicted by ART and reap the permanent benefits from this promising technology.

\section{References}

1. Sharpe K, Karovitch AJ, Claman P, Suh KN. Transvaginal oocyte retrieval for in vitro fertilization complicated by ovarian abscess during pregnancy. Fertil Steril. 2006 Jul;86(1):219.e11-3.

2. Matsunaga Y, Fukushima K, Nozaki M, Nakanami N, Kawano Y, Shigematsu T, Satoh S, Nakano H. A case of pregnancy complicated by the development of a tuboovarian abscess following in vitro fertilization and embryo transfer. Am J Perinatol. 2003 Aug;20(6):277-82.

3. Ben Saad M, Attia L, Ben Temime R, Kilani M, Makhlouf T, Chachia A, Koubâa A. Ovarian abscess as a complication of assisted reproduction techniques: a case report.Tunis Med. 2010 Apr;88(4):285-7.

4. Moini A, Riazi K, Amid V, Ashrafi M, Tehraninejad E, Madani T, Owj M. Endometriosis may contribute to oocyte retrieval-induced pelvic inflammatory disease: report of eight cases. J Assist Reprod Genet. 2005
Aug;22(7-8):307-9.

5. Jakimiuk AJ, Grzybowski W, Zakrzewski J, Lewandowski P, Kozioł K..[The ovarian hyperstimulation syndrome--diagnostic criteria, management procedures][Article in Polish]. Ginekol Pol. 2006 Nov;77(11):885-92

6. Aboulghar M. Symposium: Update on prediction and management of OHSS. Prevention of OHSS. Reprod Biomed Online. 2009 Jul;19(1):33-42.

7. Chang Y, Lee JN, Yang CH, Hsu SC, Tsai EM.An unexpected quadruplet heterotopic pregnancy after bilateral salpingectomy and replacement of three embryos. Fertil Steril. 2003 Jul;80(1):218-20.

8. Chin HY, Chen FP, Wang CJ, Shui LT, Liu YH, Soong YK.Heterotopic pregnancy after in vitro fertilization-embryo transfer. Int J Gynaecol Obstet. 2004 Sep;86(3):4116.

9. Allen VM, Wilson RD, Cheung A; Genetics Committee of the Society of Obstetricians and Gynaecologists of Canada (SOGC); Reproductive Endocrinology Infertility Committee of the Society of Obstetricians and Gynaecologists of Canada (SOGC).Pregnancy outcomes after assisted reproductive technology.[Article in English, French] J Obstet Gynaecol Can. 2006 Mar;28(3):220-50.

10. Grandone E, Colaizzo D, Vergura P, Cappucci F, Vecchione G, Lo Bue A, Cittadini E, Margaglione M. Age and homocysteine plasma levels are risk factors for thrombotic complications after ovarian stimulation. Hum Reprod. 2004 Aug;19(8):1796-9.

11. Pappo I, Lerner-Geva L, Halevy A, Olmer L, Friedler S, Raziel A, Schachter M, Ron-El R. The possible association between IVF and breast cancer incidence. Ann Surg Oncol. 2008 Apr;15(4):1048-55.

12. Brinton L. Long-term effects of ovulation-stimulating drugs on cancer risk. Reprod Biomed Online. 2007 Jul;15(1):38-44. 